\title{
Near-Capacity Multi-Layered Code Design for LACO-OFDM-Aided Optical Wireless Systems
}

\author{
Zunaira Babar, Xiaoyu Zhang, Panagiotis Botsinis, Dimitrios Alanis, \\ Daryus Chandra, Soon Xin Ng and Lajos Hanzo
}

\begin{abstract}
In this letter, we conceive a multi-layered coding scheme for the recently proposed Layered Asymmetrically Clipped Optical Orthogonal Frequency Division Multiplexing (LACO-OFDM) system, which exploits its layered structure for protecting the information bits transmitted in the base layer. Explicitly, we intrinsically integrate error correction into the layered structure of the LACO-OFDM system and invoke soft iterative decoding between the layers at the receiver. Our system operates within $2 \mathrm{~dB}$ of the capacity limit at a BER of $10^{-4}$.
\end{abstract}

Keywords-Layered ACO-OFDM, Iterative Decoding, MultiLayered Coding.

\section{INTRODUCTION}

Over the recent years, there has been growing interest in optical wireless powered Vehicle-to-Vehicle (V2V) and Vehicle-to-Infrastructure (V2I) communication systems [1], [2]. Furthermore, the data rate requirements are anticipated to increase tremendously by 2020 [3]. In this context, Layered Asymmetrically Clipped Optical Orthogonal Frequency Division Multiplexing (LACO-OFDM) [4] is a promising candidate for transmission by virtue of its high power and spectral efficiency. Explicitly, the conventional Asymmetrically Clipped Optical OFDM (ACO-OFDM) [5] only utilizes the odd-indexed subcarriers for transmission. By contrast, LACOOFDM embeds multiple ACO-OFDM layers within the classic ACO-OFDM frame. These additional ACO-OFDM layers occupy the blank even-indexed subcarriers; hence achieving a higher spectral efficiency.

The specific frame structure of LACO-OFDM ensures that the Inter-Layer Interference (ILI) of lower layers contaminates only the upper layers. Consequently, the distinct ACO-OFDM layers can be sequentially detected, starting from the lowest layer, which is free from ILI. The higher layers are then detected by subtracting the ILI of lower layers, which may be estimated based on the detected uncontaminated base-layer symbols after remodulating them. Unfortunately, any error encountered in the detection of lower layers propagates to all subsequent layers. Consequently, higher layers experience a higher Bit Error Ratio (BER) [4], [6]. An iterative detection method was conceived in [7] for reducing the ILI. Later, soft Successive Interference Cancellation (SIC) based detection

The authors are with the School of Electronics and Computer Science, University of Southampton, SO17 1BJ, United Kingdom. Email: \{zb2g10, xz3u13, dc2n14, sxn, lh\}@ecs.soton.ac.uk.

This work was supported in part by the European Research Council through the Advanced Fellow Award QuantCom and in part by the Engineering and Physical Sciences Research Council under Grant EP/PO34284/1. was proposed in [8], which was shown to completely eliminate ILI. Furthermore, a diversity combing module was also introduced in the receiver of LACO-OFDM in [8]. Diversity combining in LACO-OFDM was further explored in [9], where both frequency domain and time domain diversity were independently explored.

Zhang et al. [10] were the first to integrate Forward Error Correction (FEC) codes into the LACO-OFDM system. It was shown in [10] that, for the sake of reducing ILI in a coded LACO-OFDM system, it is more beneficial to embed FEC codes in each layer rather than using a single FEC code serially concatenated with the LACO-OFDM module. The resulting coding scheme of [10] is named 'multi-class coded LACOOFDM'. Analogous to the uncoded LACO-OFDM system, the higher layers of a multi-class coded LACO-OFDM scheme exhibit a worse BER than the lower layers. Hence, each layer offers a different level of protection, which is generally not desirable unless the system is used for unequal-protection joint source-and-channel coding [11]. Ideally, all layers should offer the same level of protection. In this spirit, we conceive an iterative multi-layered code design in this paper, which ensures that the system offers the same level of protection for all information bits without compromising the net achievable performance. Explicitly, we exploit the additional LACO layers for transmitting the redundant bits of the FEC; hence, spreading redundancy across the layers. It is demonstrated that the designed system operates within $2 \mathrm{~dB}$ of the capacity limit at a BER of $10^{-4}$.

This letter is structured as follows. In Section II, we detail the multi-layered coded LACO-OFDM system. Explicitly, we lay out the system model of the transmitter in Section II-A, while the receiver is described in Section II-B. We next discuss our results in Section III, while our conclusions are offered in Section IV.

\section{Multi-Layered Coded LACO-OFDM}

\section{A. Transmitter}

Inspired by the multiple-component turbo codes relying on Unity Rate Codes (URC) [12], [13], which are known to outperform the classic twin-component turbo codes, we conceive the multi-layered coded LACO-OFDM scheme of Fig. 1. In the multi-class coded LACO-OFDM of [10], each layer encodes a different information stream. By contrast, the proposed multi-layered coded LACO-OFDM encodes the same information in each layer. Hence, while the multi-class coded LACO-OFDM of [10] spreads redundancy within a 


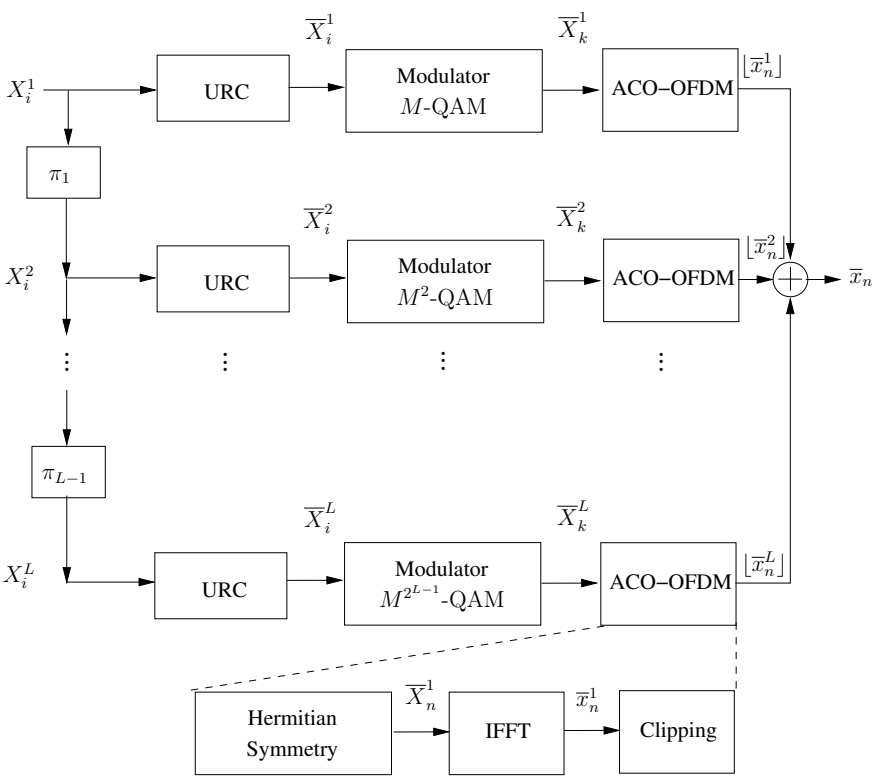

Fig. 1: Schematic of multi-layered coded LACO-OFDM transmitter, having $L \leq \log _{2} N$ LACO layers, where $N$ denotes the number of subcarriers.

layer, the multi-layered coded LACO-OFDM of Fig. 1 spreads redundancy across the layers in the spirit of invoking iterations between the LACO-OFDM layers. This in turn ensures that all information bits experience the same BER.

At the transmitter, the information stream $X_{i}^{1}$ is fed to the first layer, while its bit-interleaved versions $\left\{X_{i}^{2}, X_{i}^{3}, \ldots, X_{i}^{L}\right\}$ are fed to the subsequent layers, as demonstrated in Fig. 1. It is important to mention here that the same interleaver can be used for all the layers without compromising the achievable performance [12], [13]. Each layer is then independently encoded using a URC. The resultant encoded bit stream $\bar{X}_{i}^{l}$ of the lth layer is modulated using $M^{2^{l-1}}$-QAM, yielding the complex valued symbols $\bar{X}_{k}^{l}$, where $0 \leq k \leq\left(N / 2^{l+1}-1\right)$ given that we have $N$ subcarriers. Hence, each layer yields $N / 2^{l+1}$ complex-valued symbols, which are independently fed to the ACO-OFDM module of Fig. 1. It may be noticed here that the number of symbols reduces by half for each successive layer. However, since we are proportionally increasing the number modulation levels of the QAM modulator, we ensure that each layer transmits the same number of information bits as the first layer. Hence, the multi-layered coded LACO-OFDM of Fig. 1 transmits $N\left(\log _{2} M\right) / 4$ information bits using $N$ subcarriers and the resultant transmission efficiency is $\left(\log _{2} M\right) / 4$ information bits per subcarrier.

The ACO-OFDM module of Fig. 1 modulates the complexvalued symbols of the first layer $(l=1)$ onto the odd subcarriers to ensure that the time-domain signal can be clipped at zero without losing information. Furthermore, Hermitian symmetry is imposed to guarantee a real-valued time-domain signal. The resultant frequency-domain ACO-OFDM signal of the first layer is given by:

$$
\bar{X}^{1}=\left[0, \bar{X}_{0}^{1}, 0, \bar{X}_{1}^{1}, 0, \ldots, \bar{X}_{N / 4-1}^{1}, 0, \bar{X}_{N / 4-1}^{1 *}, 0, \ldots, \bar{X}_{0}^{1 *}\right],
$$

where $\left[{ }^{*}\right]$ denotes the complex conjugate operation and 0 represents the blank even-indexed subcarriers. The signal of Eq. (1) is equivalent to that of a classic ACO-OFDM signal, which utilizes only $1 / 4$ th of the available bandwidth. LACOOFDM enhances the system's spectral efficiency by utilizing the blank (zero-valued) subcarriers of Eq. (1) for transmitting the symbols of the the subsequent layers. More precisely, the frequency-domain signal of the $l$ th layer is equivalent to:

$$
\bar{X}_{n}^{l}= \begin{cases}\bar{X}_{k}^{l}, & \text { if } n=2^{l} k+2^{l-1}, \\ \bar{X}_{k}^{l *}, & \text { if } n=N-\left(2^{l} k+2^{l-1}\right), \\ 0, & \text { otherwise, }\end{cases}
$$

for $0 \leq n \leq(N-1)$, which ensures that the $N / 2^{l}$ nonzero symbols of each layer are transmitted using orthogonal subcarriers.

The frequency domain signal of each layer is independently passed through an $N$-point Inverse FFT (IFFT) to obtain the time-domain signal $\bar{x}_{n}^{l}$, which can be expressed as:

$$
\bar{x}_{n}^{l}=\frac{1}{\sqrt{N}} \sum_{z=0}^{N-1} \bar{X}_{z}^{l} \exp \left(\frac{j 2 \pi n z}{N}\right) .
$$

The time-domain signal of Eq. (3) is anti-symmetric with respect to the $\mathrm{x}$-axis, so that we have $\bar{x}_{n}^{l}=-\bar{x}_{n+N / 2^{l}}^{l}$. Consequently, it can be clipped at zero without information loss, yielding the all-positive signal $\left\lfloor\bar{x}_{n}^{l}\right\rfloor$ modeled as:

$$
\left\lfloor\bar{x}_{n}^{l}\right\rfloor= \begin{cases}\bar{x}_{n}^{l}, & \text { if } \bar{x}_{n}^{l} \geq 0, \\ 0, & \text { if } \bar{x}_{n}^{l}<0 .\end{cases}
$$

This negative clipping process introduces distortion in the frequency domain, which appears on the blank subcarriers of the $l$ th layer occupied by the higher layers, i.e. layers $(l+1)$ to $L$. Finally, the clipped time-domain signals of all the $L$ layers are superimposed on each other for simultaneous transmission via LEDs, i.e. we have:

$$
\left\lfloor\bar{x}_{n}\right\rfloor=\sum_{l=1}^{L}\left\lfloor\bar{x}_{n}^{l}\right\rfloor .
$$

In the frequency domain, this is equivalent to superimposing the information symbols and the negative clipping distortion, which appear on the same subcarrier. More specifically, the clipping distortion of all the lower layers, i.e. layers 1 to $(l-1)$, interferes with the information symbols of the $l$ th layer $(l>1)$; hence, resulting in ILI.

\section{B. Receiver}

Fig. 2 shows the schematic of the proposed multi-layered coded LACO-OFDM receiver, which is discussed below.

At the receiver, the optical signal is detected by an avalanche photodiode, which converts the received optical signal to an 


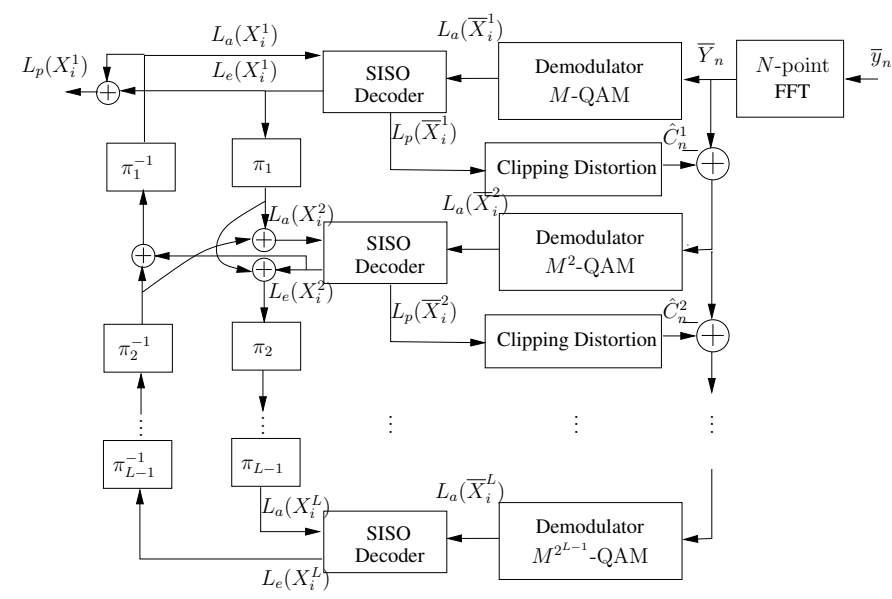

Fig. 2: Schematic of multi-layered coded LACO-OFDM receiver, having $L \leq \log _{2} N$ LACO layers, where $N$ denotes the number of subcarriers.

electronic signal. Assuming that the thermal and shot noise experienced by the electronic signal can be modeled as Additive White Gaussian Noise (AWGN) $w_{n}$ having a mean of zero and a variance of $\sigma^{2}$, the noisy received signal can be represented as:

$$
\bar{y}_{n}=\left\lfloor\bar{x}_{n}\right\rfloor+w_{n} .
$$

The received signal of Eq. (6) is then fed to the FFT block of Fig. 2, which generates the corresponding frequency-domain signal $\bar{Y}_{n}$ given by:

$$
\bar{Y}_{n}=\frac{1}{2} \sum_{l=1}^{L}\left(\bar{X}_{n}^{l}+C_{n}^{l}\right)+W_{n},
$$

where $C_{n}^{l}$ denotes the clipping noise of the $l$ th layer, while $W_{n}$ is the FFT of AWGN noise. Recall that the lth layer experiences ILI from all the layers 1 to $(l-1)$. Consequently, the ILI from lower layers must be subtracted from the $l$ th layer for the sake of accurately estimating the information symbols. In line with the LACO-OFDM detector of [4], we achieve this by commencing detection from the first layer, which is free from clipping distortion. Explicitly, the oddindexed subcarriers of $\bar{Y}_{n}$, i.e. $n=[1,3, \ldots N / 2-1]$, are fed to the $M$-QAM demodulator, which yields the soft a-priori Log-Likelihood Ratios (LLRs) $L_{a}\left(\bar{X}_{i}^{1}\right)$ pertaining to the URC encoded bits $\bar{X}_{i}^{1}$. The a-priori encoded LLRs $L_{a}\left(\bar{X}_{i}^{1}\right)$ and the a-priori uncoded LLRs $L_{a}\left(X_{i}^{1}\right)$ received from the other constituent SISO decoders (initialized to zero for the first iteration) are then combined by the Soft-In Soft-Out (SISO) decoder of the first layer of Fig. 2 to obtain the extrinsic uncoded LLRs $L_{e}\left(X_{i}^{1}\right)$ and the a-posteriori encoded LLRs $L_{p}\left(\bar{X}_{i}^{1}\right)$. The extrinsic uncoded LLRs $L_{e}\left(X_{i}^{1}\right)$ are interleaved and added to the de-interleaved extrinsic uncoded LLRs received from the SISO decoders of the higher layers to obtain the a-priori uncoded LLRs $L_{a}\left(X_{i}^{2}\right)$ for the second layer. Please note that the extrinsic uncoded LLRs received from the higher layers are initialized to zero for the first iteration. Furthermore, as can be seen in Fig. 2, the a-posteriori encoded LLRs $L_{p}\left(\bar{X}_{i}^{1}\right)$ are fed to the 'Clipping Distortion' block for estimating the frequency-domain clipping distortion of the first layer $C_{n}^{l}$, which interferes with the information symbols of the higher layers. This estimation proceeds as follows:

- URC encoded bits of the first layer are estimated by making hard-decision based on the a-posteriori encoded LLRs $L_{p}\left(\bar{X}_{i}^{1}\right)$. Let us denote the estimated bits as $\hat{\bar{X}}_{i}^{1}$.

- The estimated bits $\hat{\bar{X}}_{i}^{1}$ are modulated using the $M$-QAM modulator and passed through the ACO-OFDM module, which imposes Hermitian symmetry and IFFT as well as clipping analogous to the transmitter of Fig. 1. This yields the estimated time-domain signal $\left\lfloor\hat{\bar{x}}_{n}^{1}\right\rfloor$.

- Finally, the FFT of $\left\lfloor\hat{x}_{n}^{1}\right\rfloor$ is carried out to estimate the negative clipping distortion falling on the even-indexed subcarriers, denoted as $\hat{C}_{n}^{1}$.

The estimated negative clipping distortion $\hat{C}_{n}^{1}$ is subtracted from the received signal $\bar{Y}_{n}$ and the resultant signal is fed to the $M^{2}$-QAM demodulator of the second layer. Explicitly, since the information symbols of the $l$ th layer are located at $n=2^{l} k+2^{l-1}$ for $0 \leq k \leq\left(N / 2^{l+1}-1\right)$, according to Eq. (2), only the $n$th indexed subcarriers are fed to the QAM demodulator. The above process is repeated for all the subsequent layers, as illustrated in Fig. 2. The $L$ constituent SISO decoders of Fig. 2 engage in iterative decoding until the maximum number of decoding iterations is reached.

\section{RESUlTS AND Discussions}

For the sake of quantifying the performance of the proposed multi-layered LACO scheme, we consider a 3-layer LACO-OFDM system, invoking BPSK modulation for the first layer and relying on binary memory-1 URCs, having the feedback polynomial $(2,3)_{\text {Octal }}$. Based on the system model of Fig. 1, 4-QAM and 16-QAM are used for the second and third layers, respectively, and the resultant transmission efficiency is 0.25 information bits per subcarrier. Furthermore, we ensure that the average symbol power (electrical power) is normalized to unity for all modulation schemes. The resultant BER performance curves after $1,2,5$ and 10 iterations are recorded in Fig. 3 for $N=2048$ subcarriers and interleavers of length 4096 (equivalent to 8 LACO-OFDM frames). We may observe in Fig. 3 that the performance improves upon increasing the number of iterations. However, this happens with diminishing returns. As a benchmark, we have marked the corresponding Discrete-input Continuous-output Memoryless Channel (DCMC) capacity [10] in Fig. 3. Quantitatively, at a BER of $10^{-4}$, the performance of the proposed multi-layered LACO-OFDM system is within $2 \mathrm{~dB}$ of the DCMC capacity after 15 iterations.

As another benchmark, we also compare our system to the multi-class coded LACO-OFDM system of [10], which independently encodes and decodes the information in each layer. Our benchmark scheme invokes a $1 / 2$-rate code for 


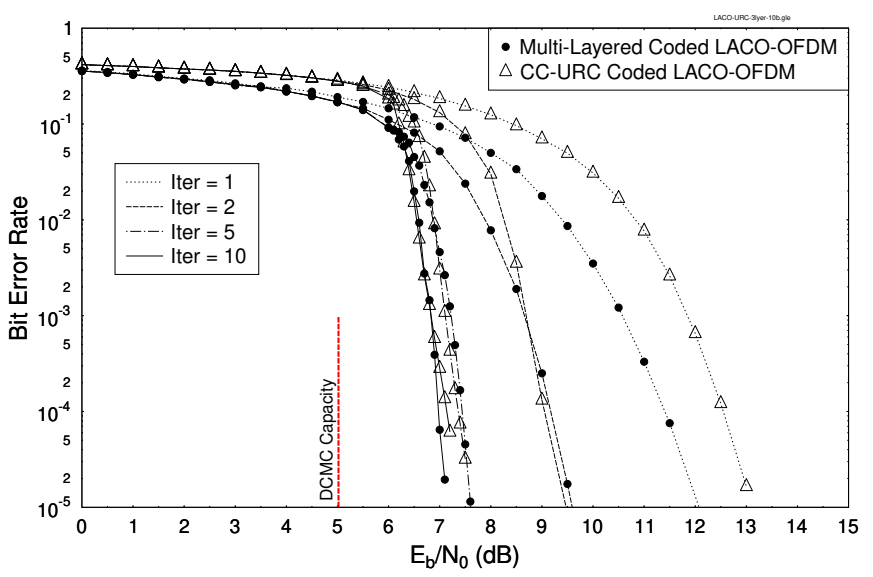

Fig. 3: Achievable BER performance of 3-layer multi-layered coded LACO-OFDM system of Fig. 1, having $M=2$ and binary memory- 1 URCs. We have used $N=2048$ subcarriers and interleavers of length 4096 (equivalent to 8 LACO-OFDM frames). We have benchmark the performance against the DCMC capacity as well as the multi-class coded LACOOFDM system of [10] relying on CC-URC codes.

each of the three layers and transmits using BPSK modulation, which results in a transmission efficiency of 0.21875 . For the sake of ensuring that the decoding complexity of the two schemes is comparable, we use iterative 1/2-rate codes in the benchmark scheme, so that both schemes invoke the same number of trellis states in each decoding iteration. Explicitly, our multi-layered coded LACO-OFDM system of Fig. 3 relies on $\left(2^{1} \times 3\right)=6$ trellis states in each iteration, since three memory-1 URCs are activated in each iteration. To match this decoding complexity, the benchmark multi-class coded scheme invokes the same iterative CC-URC coding scheme for each layer, which consists of a serially-concatenated memory2 1/2-rate convolutional code and a memory-1 URC. We may observe in Fig. 3 that the proposed multi-layered coded LACOOFDM system initially outperforms the multi-class coded LACO-OFDM system of [10]. However, the performance of the two systems becomes comparable upon increasing the number of iterations. Nonetheless, the multi-layered coded LACO-OFDM system brings with it the benefit of offering the same level of protection for all information bits. By contrast, the BER of the multi-class coded LACO-OFDM system of [10] is not the same for all layers, as demonstrated in Fig. 4. More precisely, the higher layers of the multi-class coded LACOOFDM system of [10] experience a significantly higher BER than the lower layers. Hence, the multi-class coded LACOOFDM system of [10] is not suitable for applications, where the same level of robustness is desired for all information bits. However, this uniform BER is achieved at the cost of additional FFT/IFFT operations. Recall from Section II-B that a pair of IFFT and FFT operations is used for estimating the clipping distortion of the lth layer. The multi-class coded LACO-OFDM of [10] estimates the clipping distortion only once for every

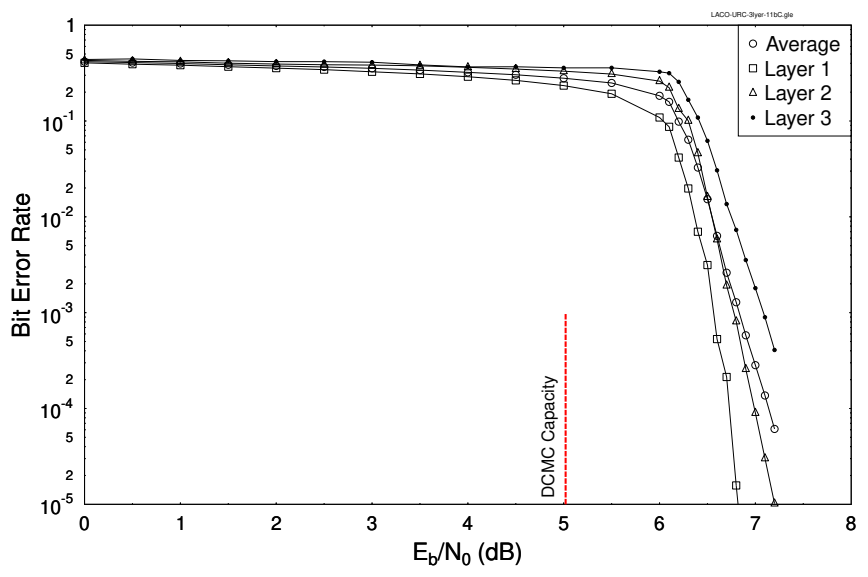

Fig. 4: Achievable BER performance of each layer after 10 iterations for the benchmark scheme CC-URC of Fig. 3.

layer during the entire decoding process. By contrast, our proposed scheme of Fig. 2 estimates the clipping distortion once for every layer during each decoding iteration. Hence, the number of IFFT/FFT operations invoked is proportional to the number of decoding iterations.

\section{CONCLUSIONS}

We have conceived a multi-layered coding scheme for the LACO-OFDM system invoking binary memory-1 URCs. Explicitly, the higher layers of the LACO-OFDM system are utilized to send redundant information pertaining to the information bits of the base layer. At the decoder, the multiple LACO-OFDM layers engage in soft iterative decoding for recovering the information bits of the base layer. We have benchmarked the performance of the resultant system both against the achievable DCMC capacity as well a comparable coding scheme, which transmits redundancy within the layers.

\section{REFERENCES}

[1] J. Jiang, P. Zhang, R. Zhang, S. Chen, and L. Hanzo, "Aperture selection for ACO-OFDM in free-space optical turbulence channel," IEEE Transactions on Vehicular Technology, vol. 65, no. 8, pp. 60896100, 2016.

[2] A.-M. Căilean and M. Dimian, "Current challenges for visible light communications usage in vehicle applications: A survey," IEEE Communications Surveys \& Tutorials, vol. 19, no. 4, pp. 2681-2703, 2017.

[3] H. Huang, J. Wang, J. Wang, J. Yang, J. Xiong, and G. Gui, "Symbol error rate performance analysis of non-orthogonal multiple access for visible light communications," China Communications, vol. 14, no. 12, pp. 153-161, 2017.

[4] Q. Wang, C. Qian, X. Guo, Z. Wang, D. G. Cunningham, and I. H. White, "Layered ACO-OFDM for intensity-modulated direct-detection optical wireless transmission," Optics Express, vol. 23, no. 9, pp. 12 382-12393, 2015.

[5] J. Armstrong and A. Lowery, "Power efficient optical OFDM," Electronics Letters, vol. 42, no. 6, pp. 370-372, 2006.

[6] X. Zhang, Q. Wang, R. Zhang, S. Chen, and L. Hanzo, "Performance analysis of layered ACO-OFDM," IEEE Access, vol. 5, pp. 18366$18381,2017$. 
[7] Q. Wang, Z. Wang, X. Guo, and L. Dai, "Improved receiver design for layered ACO-OFDM in optical wireless communications," IEEE Photon. Technol. Lett., vol. 28, no. 3, pp. 319-322, 2016.

[8] T. Q. Wang, H. Li, and X. Huang, "Diversity combining for layered asymmetrically clipped optical OFDM using soft successive interference cancellation," IEEE Communications Letters, 2017.

[9] M. M. Mohammed, C. He, and J. Armstrong, "Diversity combining in layered asymmetrically clipped optical OFDM," Journal of Lightwave Technology, vol. 35, no. 11, pp. 2078-2085, 2017.

[10] X. Zhang, Z. Babar, R. Zhang, S. Chen, and L. Hanzo, "Multiclass coded layered asymmetrically clipped optical OFDM," IEEE Transactions on Communications, 2018.

[11] L. Hanzo, T. H. Liew, B. L. Yeap, R. Y. S. Tee and S. X. Ng, Turbo Coding, Turbo Equalisation and Space-Time Coding: EXIT-Chart-Aided Near-Capacity Designs for Wireless Channels, 2nd Edition. New York, USA: John Wiley IEEE Press, March 2011.

[12] H. Chen, R. G. Maunder, and L. Hanzo, "An EXIT-chart aided design procedure for near-capacity N-component parallel concatenated codes," in Global Telecommunications Conference (GLOBECOM 2010), 2010 IEEE. IEEE, 2010, pp. 1-5.

[13] — "Low-complexity multiple-component turbo-decoding-aided hybrid ARQ," IEEE Transactions on Vehicular Technology, vol. 60, no. 4, pp. 1571-1577, 2011. 